\title{
SUMMARY OF THE HEAVY ION PHYSICS SESSIONS AT LAKE LOUISE
}

John W. Harris

\author{
Nuclear Science Division \\ Lawrence Berkeley Laboratory \\ University of California \\ Berkeley. California 94720
}

\section{DISCLAIMER}

\begin{abstract}
This report was prepared as an account of work sponsored by an agency of the United States Government. Neither the United States Government nor any agency thereof, nor any of their employees, makes any warranty, express or implied, or assumes any legal liability or responsibility for the accuracy, completeness, or usefulness of any information, apparatus, product, or process disclosed, or represents that its use would not iníringe privately owned rights. Reference herein to any specific commercial product, process, or service by trade name, trademark, manufacturer, or otherwise does not necessarily constitute or imply its endorsement, recommendation, or favoring by the United States Government or any agency thereof. The views and opinions of authors expressed herein do not necessarily state or reflect those of the United Stuces Government or any agency thereof.
\end{abstract}

2nd Conference on the Intersections Between Particle and Nuclear Physics, Lake Louise, Canada, May 25-31, 1986

This work was supported by the Director, Office of Energy Research, Division of Nuclear Physics of the Office of High Energy and Nuclear Physics of the U.S. Department of Energy under Contract OE-AC03-76SF00098. 


\title{
SUMMARY OF THE HEAVY ION PHYSICS SESSIONS AT LAKE LOUISE
}

\author{
John W. Harris \\ Nuclear Science Division, Lawrence Berkeley Laboratory \\ University of California, Berkeley, CA 94720 USA
}

In the last several years the intersections between particle and nuclear physics have grown rapidly to the point where many particle a I nuclear physicists now speak the same language and even work together on the same experiments. In heavy ion physics this interdisciplinary communication ranges from describing ground state nuclei and highly excited nuclear matter in terms of quark degrees of freedom to searching for quark deconfinemeni in high energy nucleus-nucleus interactions utilizing nuclear beams from high energy physics accelerators. Presentations in the Heavy lon Physics Sessions encompass this range and can for the most part be divided into two subcategories. The first is the study of different phases of nuclear matter. This typically refers to understanding the nuclear equation of state from low taryon densities and temperatures (where a liquid-gas phase transition should cacur) through nuclear structure, neutron star formation and supernova explosi in to the high densities and temperatures where the onset of deconfineme? $t$ of quarks in nuclear matter is predicted. Beyond this is the second region, that of the study of quark matter.

In the quest to understand the nuclear equation of state considerable advances have been made on several fronts. A semi-empirical equation of state has been extracted from pion production data in the Streamer Chamber ${ }^{1}$ in relativistic nucleus-nucleus collisions at the Bevalac. This is shown in Fig.1 along with theoretical equations of state labelled FP, 2 BCK, 3 and VUU 4 which are used in nuclear matter calculations, supernova simulations and microscopic calculations for relativistic nucleus-nucleus collisions, respectively. The FP and BCK equations of state are considerably "softer" than that extracted from the data and VUU. This apparent discrepancy is assuaged by the fact that relativistic nucleus-nucleus collisions, supernova explosions and nuclear structure effects are governed by the nuclear equation of state in different regions of temperature and density. Nuclear structure studies and nuclear matter calculations near the ground state of nuclei reflect the equation of state at zero temperature near saturation density po while neutron star formation and supernova explosions are govemed by the nuclear equation of state at low temperatures $(T \sim 15 \mathrm{MeV})$ and densities $p \sim 2 p 0$. In contrast relativistic nucleus-nucleus collisions are sensitive to the nuclear equation of state at high temperatures ( $T-60-100 \mathrm{MeV}$ ) and densities $p \sim 3-4 p 0$. The behavior of the nuclear equation of state under these different conditions has yet to be fully understood. However, it should be stiffer at higher temperatures partly due to a decrease in the effective nucleon mass and an increase in prominence of the repulsive u-interactions at high temperatures.

Another important result from the Bevalac is the recent observation and systematic study of collective sidewards flow of matter. Shown in Fig.2 are 
Equation of State of Nuclear Matter

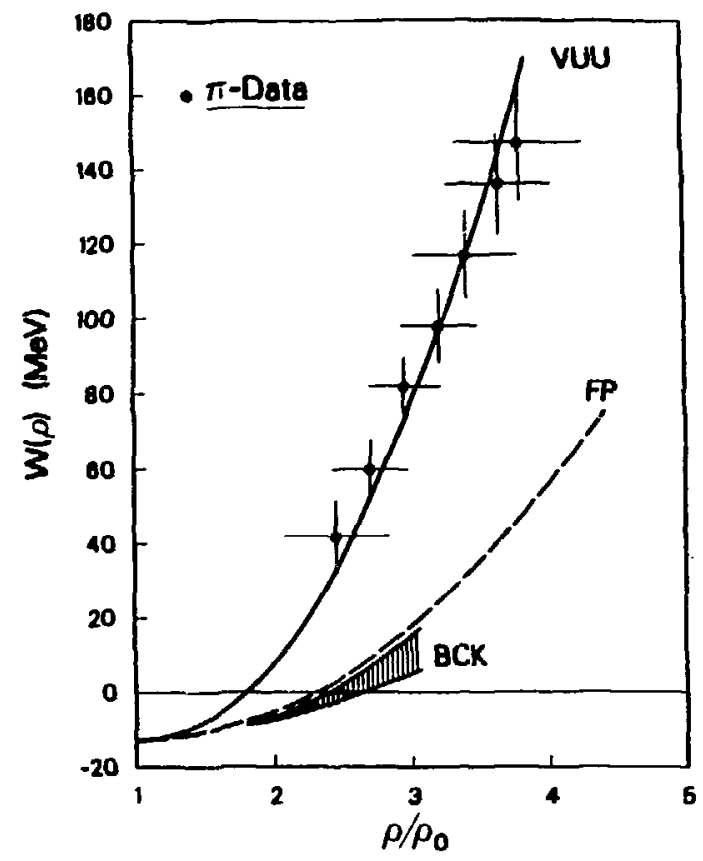

Fig.1. Internal energy per baryon as a function of nuclear density extracted trom pion multiplicity data (points). Also displayed are three theoretical equations of state (curves). See text for details.

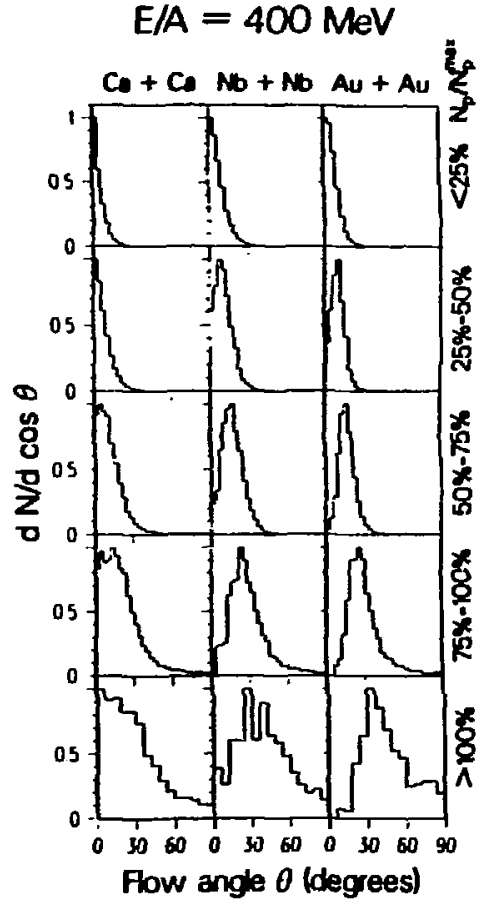

Fig.2. Flow angislar distributions for three systems as a tunction of the fractional muliiplicity. 
results from the Plastic Ball5 for three different systems at fixed incident energy. Plotted as a function of fractional multiplicity $N_{p} / N_{p} \max$ (which is inversely related to the impact parameter) are the distribution of flow angles, defined as the angle of the major axis of the event ellipsoid with respect to the beam axis, as determined from a sphericity analysis. The flow angle is observed to increase with the mass of the system and the multiplicity. These data provide strong evidence for the collective flow of matter. A rather stiff nuclear equation of state is necessary to describe these and other flow data 6 along with the pion production data.

An interesting result 7 from the Bevalac and possibly another approach 8 to the equation of state is the recent study of subthreshold kaon production. Whereas the threshold for associated production of $K^{-}$in nucleon-nucleon reactions occurs at $E_{\text {lab }}\left(\mathrm{NN}^{\prime}>\mathrm{NNK}^{+} \mathrm{OK}^{-}\right)=2.5 \mathrm{GeV}, \mathrm{K}^{-1} \mathrm{~s}$ have been observed in $\mathrm{Si}+\mathrm{Si}$ collisions at incident energies as low as $\mathrm{E}_{\mathrm{lab}}=1.3 \mathrm{GeV} / \mathrm{n}$. In an atiempt to understand the production mechanism, predictions have recently been made for the $\mathrm{K}^{-}$yields assuming various theoretical models. An estimate assuming thermal and chemical equilibrium, which is unreasonable below threshold, overpredicts the yields by a factor of 20 as would be expected. Quite surprisingly, a single collision Fermi momentum model ${ }^{9}$ underpredicts the yield by a factor of 20 . Two more sophisticated microscopic models, the hadrochemical model 10 and semianalytic transport theory ${ }^{11}$ predict the energy dependence of the data but not the correct magnitude of the $K^{-}$yields or all the systematics observed in the data. These models and experiments are still somewhat in their infancy. However, subthreshold kaon production, which should be more sensitive than pion production to the available energy and in turn to the compressional degrees of freedom, may provide new information on the nuclear equation of state as the experimental techniques become more refined and the models better developed.

On the way to studying the properties of nuclear collisions in the quark mattar regime, several interesting experiments have already taken place. In $\mathrm{T}$. Ludlam's talk 12 he discussed the mechanism by which gammas and dileptons should probe the early stages of relativistic nucleus-nucleus collisions, and specifically may be signatures for the formation of a quark-gluon plasma. Single leptons, namely electrons, have been measured over a large incident energy range from KEK in Japan to the ISR at CERN and the e/n ratio has been found to be relatively independent of projectile-target-energy combinations. 13 Experiments on the lepton and dilepton production in Pp, DA and AA reactions are underway 13 at the Bevalac and should provide valuable and necessary information for understanding the lepton production in future searches for the quark-gluon plasma.

Results from the ISR light ion running periods have also shed first light on nuclear effects in very high energy nuclear reactions. Displayed in Fig.3 are results for the total neutral energy spectra from pp and aa by the R110-BCMOR collaboration. 14 The pp data are well fit by a single gamma function over nine orders of magnitude in cross section as represented by the solid curve. The fit 


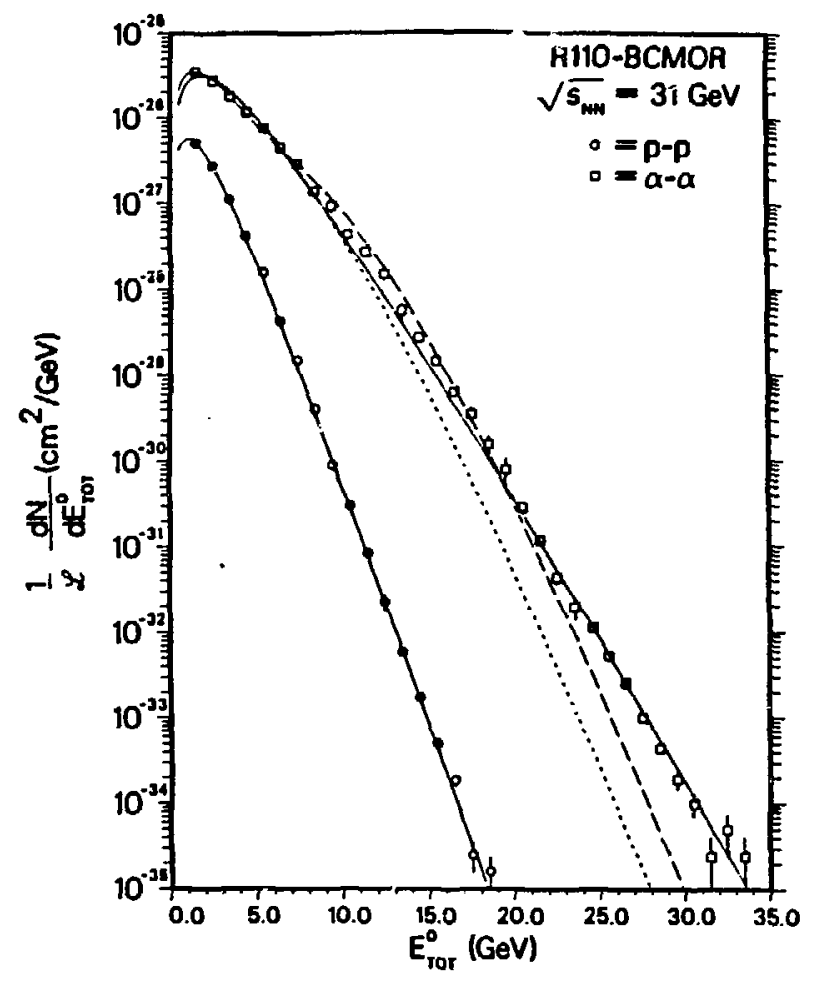

Fig.3. Total neutral energy spectra for pp and aa interactions with lits described in the text.

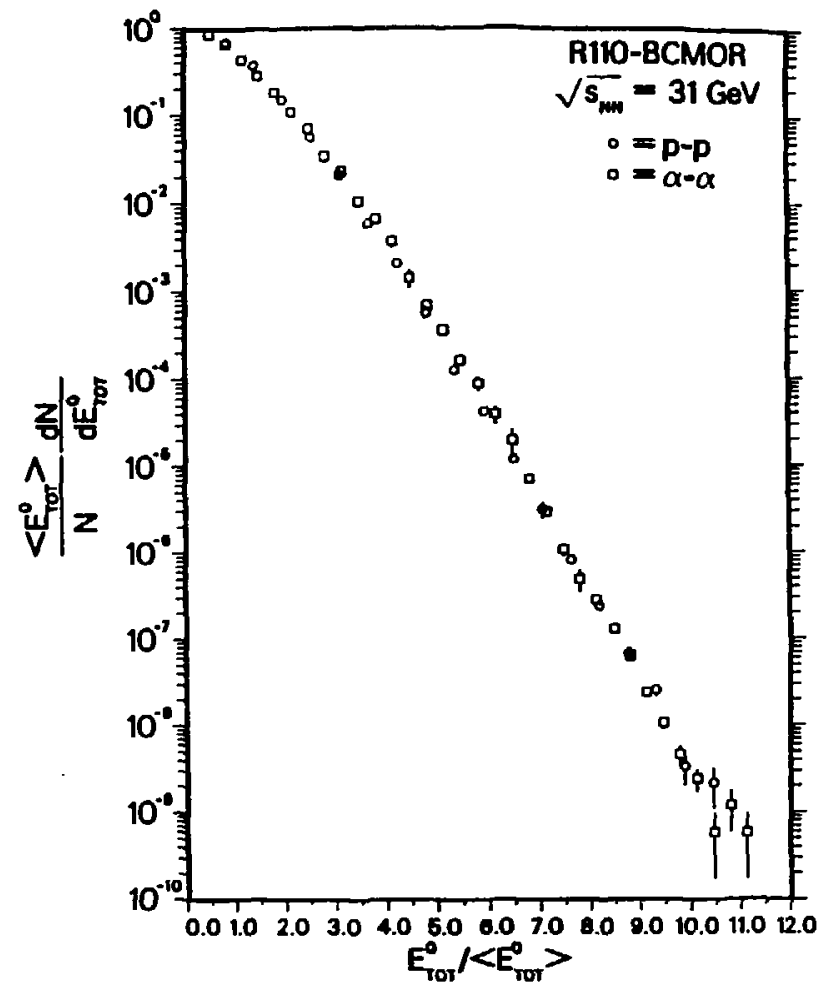

Fig.4. Total neutral energy spectra of Fig.2 plotted as a function of the scaled energy variable. 
corresponds to fitting the function $f(E)=a(a E) p-1_{\theta}-a E / \Gamma(p)$ to the data with parameters $a$ and $p$. The dotted curve on the aa data corresponds to the best fit using the n-collision probabilities derived by the AFS collaboration 15 from particle multiplicity data. This only fits the data at the lowest neutral energies. The dashed curve corresponds to the wounded nucleon model in the extreme case where all nucleons are assumed to participate in the collision. It still underpredicts the high neutral energy part of the spectrum. Only the solid curve where a gamma function was fit to the ad data with all parameters free can fit the data over the entire range of neutral energies. It was found that the parameters $p$ for the $p p$ and a cases were identical thereby obeying KNO scaling. 16 This can be seen by plotting the pp and ac data as a function of the scaling variable $E_{0}{ }^{\text {tot }} /<E_{0}{ }^{\text {tot }}>$ where $<E_{0}{ }^{\text {tot }}>$ is the average value of the total neutral energy E0tot. This is shown in Fig.4 where the pp and aa data overlay each other identically. A fundamental description for this simple phenomenon has not been presented but the results strongly suggest that nucleons in the helium nuclei are strongly correlated. A final comment on these data. The tail of the aa data above $20 \mathrm{GeV}$ (at a level of $10^{-5}$ of the interaction cross section) exhibits tremendous energy density, $15 \mathrm{GeV}$ per unit rapidity. This corresponds approximately to an energy density of $1.3 \mathrm{GeV} / \mathrm{fm}^{3}$ which is already quite near the predicted critical energy density for formation of the quark-gluon plasma.

Insight into the quark matter regime has come recently from advances in QCD lattice gauge calcula:ions. A consistent set of results appears to be emerging from calculations which employ different approximation schemes for solving finite temperature CCD on the lattice. Displayed in Fig.5 are results from three different approaches 17 each indicating two coupled, weak, first order phase transitions. Plotted as a function of $\beta=6 / g^{2}$, where $g$ is the coupling constant, are the order parameter for chiral symmetry $<\bar{\psi} \psi b$ and the quantity $\langle L$, the expectation value of the thermal Wilson line (or Polyakov loop). $\langle L\rangle$ is a measure of the change in free energy of the system. If the free energy $F$ of an isolated quark diverges, i.e. $\theta^{-F / T}=\langle L\rangle \rightarrow 0$, then a deconfinement transition appears as a sudden increase in $<L>$ to some nonzero value. This is observed in all three calculations. The urder parameter for chiral symmetry $\langle\bar{\psi} \psi b$, which is a measure of the quark-antiquark mass, is also observed to abruptly change at this same value of $\beta$ approaching zero where chiral symmetry restoration occurs. It appears that the deconfinement transition and chiral symmetry restoration may be related as they appear coincidentally. To better understand this and the effects of the various approximation schemes, more physical observables will have to be investigated through the use of faster calculations, requiring large amounts of supercomputer time (hundreds of Cray CPU hours).

Of considerble recent interest and uncategorizable in the present heavy ion physics summary of intersections between particle and nuclear physics are the positron lines observed at the GSI in heavy ion collisions. J. Greenberg ${ }^{18}$ presented the experimental results in the plenary sessions. Theoretically there is still no consistent explanation which adequately describes the systematics of the experimental measurements. ${ }^{19}$ The singles measurements rule out internal nuclear conversion ( $A^{*}>A \theta^{+} \theta^{-}$) primarily from the line width ( 


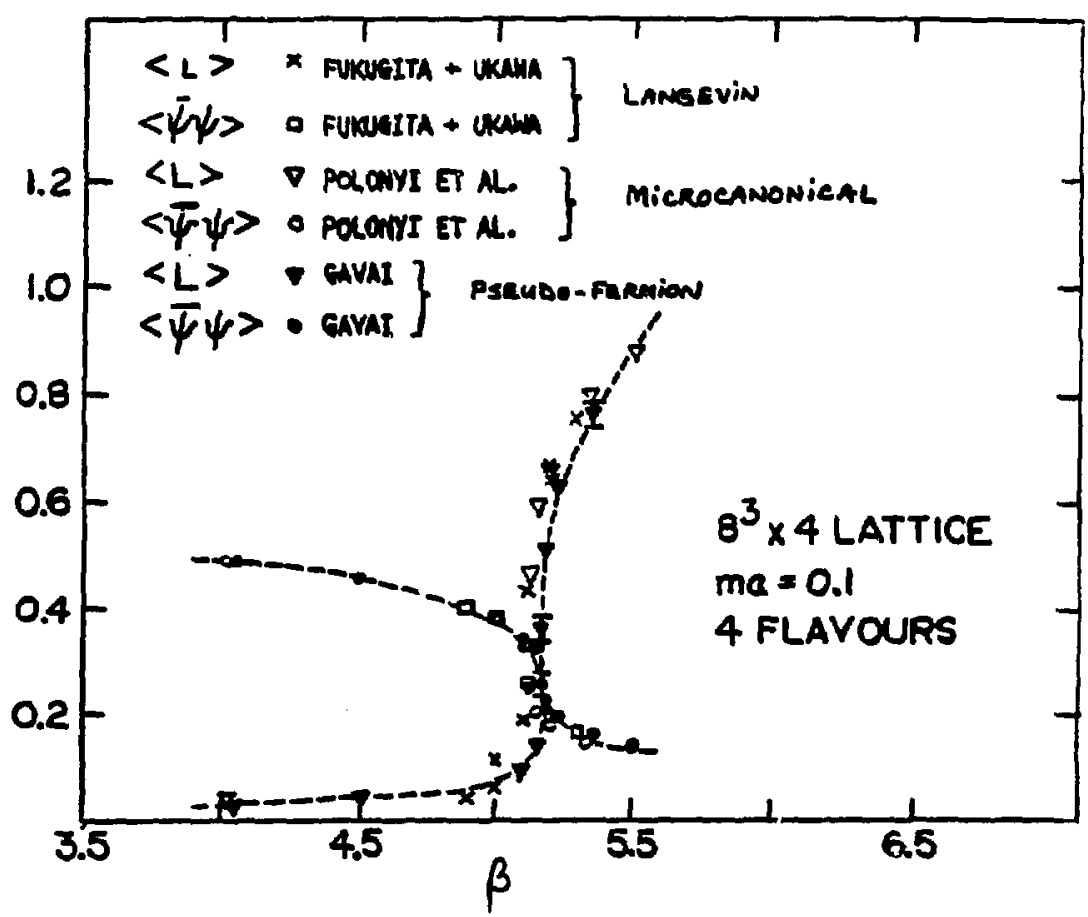

Fig.5. Expectation values of thermal Wilson line $<L>$ and chiral symmetry order parameter $<Y Y>$ plotted as a function of $B=6 / g^{2}$ from $Q C D$ lattice gauge calculations.

$3 \times 10^{-20}$ s) and gamma and electron spectra. In the coincidence measurements the narrow energy sum peak and similar energies of the $\theta^{+}$and $\theta^{-}$peaks leave open the possibility of two-body decay of a slowly moving particle ( momentum < $100 \mathrm{KeV} / \mathrm{c}$ ) in the c.m. system. The narrow energy difference peak for $\theta^{+}$and $\theta^{-}$implies decay far away from the nuclei (distance > $13000 \mathrm{~m}$ ). From the energy of the peaks, $E_{\theta+}$ and $E_{\theta-,}$ the mass of such a particle would be $M=2 m_{\theta}+E_{\theta+}+E_{\theta-}=1.78 \mathrm{MeV}$. A problem arises from the coincidence lines observed in Th + Th. At incident energy $E_{\text {lab }}=5.70 \mathrm{MeV} / \mathrm{n}$ the $\theta^{+}$and $\theta^{-}$peaks are observed at approximately $310 \mathrm{KeV}$ and at $E_{\mathrm{lab}}=5.75$ $\mathrm{MeV} / \mathrm{n}$ they appear at around $370 \mathrm{KeV}$. Is this one line that moves abruptly as the incident energy is increased slightly or are there two different lines? There still seems to be a large sentiment among experimentalists and theoreticians alike that a "simple" explanation may still loom in the future explanation of these lines, but for now there are none. The more exotic explanations 19,20 involve the decay of a pseudoscalar particle produced from the nuclear current where the prasence of multiple lines could be explained by a particle with internal structure. 
In conclusion, the intersection between particle and nuclear physics research is entering a very interesting period where in the next year there will be high energy nucleus-nucleus experiments 21 on both the European (CERNSPS) and American (BNL-AGS) continents which for the first time may have the capability to transcend the nuclear matter to quark matter barrier. Furthermore, a proposal to build a relativistic heavy ion collider (RHIC) at even higher eriergies in the US has received stre: a support from NSAC. These experiments will all involve a large number of hich energy and nuclear physicists working together to understand the common physics of nuclear and quark matter.

\section{REFERENCES}

1. R. Stock et al., Phys. Rev. Lett. 49 (1982) 1236; J.W. Harris et al., Phys. Lett. 153B (1985) 377 and J.W. Harris, "The Nuclear Matter Equation of State from Relativistic Heavy lons to Supernovae", presented at this conference.

2. B. Friedman and V.R. Pandharipande, Nucl. Phys. A351 (1981) 502.

3. E. Baron, J. Cooperstein and S. Kahana, Nucl. Phys. A440 (1985) 744.

4. H. Kruse, B.V. Jacak and H. Stöcker, Phys. Rev. Lett. 54 (1985) 289; J.J. Molitoris, D. Hahn and H. Stöcker, MSU Preprint MSUCL-530, June 1985.

5. H.G. Ritter et ai., Nucl. Phys. A447 (1985) 3c.

6. H.A. Gustafsson et al., Phys. Rev. Lett. 52 (1984) 1590; R.E. Renfordt et al., Phys Rev. Lett. 53 (1984) 763; P. Danielewicz and G. Odyniec, Phys. Lett. $157 \mathrm{~B}$ (1985) 146; D. Keane, "Collective Flow and the Stiffness of Compressed Nuclear Matter", presented at this conference; also see comparisons in J.J. Molitoris and H. Stöcker, Phys. Rev. C32 (1985) 346.

7. S. Trentalange, "Subthreshold Production of Strange Hadrons in Relativistic Heavy lon Collisions" presented at this conterence.

8. J. Aichelin and C.M. Ko, Phys. Rev. Lett. 55 (1985) 2661.

9. A. Shor et al., Phys. Rev. Lett 48 (1982) 1597.

10. H.W. Barz ot al., Z. Phys. A311 (1983) 311.

11. W. Zwermann and B. Schürmann, Phys. Lett. 145B (1984) 315.

12. T. Ludlam, "Relativistic Heavy lon Collisions" presented at this conference.

13. G. Roche, "Universality of Lepton Production in pp, pA and AA Collisions", presented at this conference.

14. A.L.S. Angelis, Phys. Lett. 168B (1986) 158 and M. Tannenbaum,

- Observation of KNO Scaling in the Neutral Energy Spectra from aa and pp Collisions at ISR Energies", presented at this conference.

15. T. Akesson et al., Phys. Lett. 119B (1982) 464.

16. Z. Koba, H.B. Nielsen and P. Olesen, Nucl. Phys. B40 (1972) 317.

17. R. Gavai, "Phase Transitions from Lattice CCD Perspectives", presented at this conference.

18. J.S. Greenberg, "Anomalous Positron Production", presented at this conference.

19. B. Muller, "Positron Lines - New Particle or Decaying Vaccum?", presented at this conference.

20. M.S. Zahir, "Production Mechanism for a Light Pseudoscalar Boson (m = 1.6 MeV) and Anomolous Positron Spectra", presented at this conference.

21. L.S. Schroeder, "Relativistic Heavy Ion Facilities", Presented at this conference. 
This report was done with support from the Department of Eneray. Any conclusions or opinions expressed in this report represent solely those of the euthor(s) and nol necessurily those of The Regents of the University of California, the Lawrence Berkeley Laboratory or the Department of Enercy.

Reference to a company or product name does not imply approval or recommendation of the product by the University of California or the U.S. Department of Energy to the exclusion of others that may be suitable. 